${ }^{8}$ D. L. Walters and C. P. Bhalla, Phys.' Rev. A 4, 2164 (1971).

${ }^{9}$ W. Bambynek, B. Crasemann, R. W. Fink, H.-U. Freund, H. Mark, C. D. Swift, R. E. Price, and P. Venugopala Rao, Rev. Mod. Phys. 44, 7.16 (1972).

${ }^{10}$ B. Crasemann, in Inner-Shell Ionization Phenomena and Future Applications, edited by R. W. Fink, S. T. Manson, J. M. Palms, and P. Venugopala Rao, U. S. Atomic Energy Commission Report No. CONF- 720404, 1973, p. 9 (unpublished).

${ }^{11}$ J. C. McGeorge, H.-U. Freund, and R. W. Fink, Nucl. Phys. A 154, 526 (1970).

${ }^{12}$ S. Mohan, H.-U. Freund, R. W. Fink, and P. Venugopala Rao, Phys. Rev. C 1, 254 (1970).

${ }^{13}$ S. Mohan, R. W. Fink, R. E. Wood, J. M. Palms, and P. Venugopala Rao, Z. Phys. 239, 423 (1970).

${ }^{14}$ J. M. Palms, R. E. Wood, P. Venugopala Rao, and V. O. Kostroun, Phys. Rev. C 2, 592 (1970).

${ }^{15}$ R. E. Wood, J. M. Palms, and P. Venugopala Rao, Phys. Rev. 187, 1497 (1969).

${ }^{16}$ P. Venugopala Rao, R. E. Wood, J. M. Palms, and R. W. Fink, Phys. Rev. 178, 1997 (1969).

${ }^{17}$ R. E. Wood, J. M. Palms, and P. Venugopala Rao, Phys. Rev. A 5, 11 (1972).

${ }^{18}$ P. Venugopala Rao, J. M. Palms, and R. E. Wood, Phys.

Rev. A 3, 1568 (1971).

${ }^{19} \mathrm{P}$. Venugopala Rao and B. Crasemann, Phys. Rev. 139, A1926 (1965).

${ }^{20}$ J. C. McGeorge, S. Mohan, and R. W. Fink, Phys. Rev. A 4, 1317 (1971).

${ }^{21}$ J. C. McGeorge and R. W. Fink, Z. Phys. 248, 208 (1971).

${ }^{22}$ J. Byrne, W. Gelletly, M. A. S. Ross, and F. Shaikh, Phys. Rev. 170, 80 (1968).

${ }^{23}$ J. Byrne, R. J. D. Beattle, S. Benda, and I. Collingwood, J. Phys. B 3, 1166 (1970).

${ }^{24}$ L. I Yin, T. Tsang, and I. Adler, in Ref. 10, p. 694.

${ }^{25}$ M. O. Krause, F. Wuilleumier, and C. W. Nestor, Jr., Phys. Rev. A 6, 871 (1972).

${ }^{26}$ L. I Yin, S. Ghose, and I. Adler, J. Geophys. Res. 77, 1360 (1972).

${ }^{27}$ L. I Yin, E. Yellin, and I. Adler, J. Appl. Phys. 42, 3595 (1971).
${ }^{28} \mathrm{~K}$. Siegbahn, D. Hammond, H. Fellner-Feldegg, and E. F. Barnett, Science 176, 245 (1972).

${ }^{29}$ K. Siegbahn, C. Nordling, G. Johansson, J. Hedman, P. F. Hedén, K. Hamrin, U. Gelius, T. Bergmark, L. O. Werme, R. Manne, and Y. Baer, ESCA Applied to Free Molecules

(North-Holland, Amsterdam, 1969), pp. 1,17.

${ }^{30}$ L. G. Parratt, Rev. Mod. Phys. 31, 616 (1959).

${ }^{31}$ K. Siegbahn, C. Nordling, A. Fahlman, R. Nordberg, K. Hamrin, J. Hedman, G. Johansson, T. Bergmark, S. Karlsson, I. Lindgren, and B. Lindberg, ESCA, Atomic, Molecular and Solid State Structure Studied by Means of Electron Spectroscopy (Almqvist and Wiksells, Uppsala, 1967).

${ }^{32}$ Y. Baer, P. F. Hedén, J. Hedman, M. Klasson, C.

Nordling, and K. Siegbahn, Phys. Scr. 1, 55 (1970).

${ }^{33}$ L. I Yin, T. Tsang, I. Adler, and E. Yellin, J. Appl. Phys. 43, 3464 (1972).

${ }^{34}$ T. W. Haas, J. T. Grant, and G. J. Dooley, Phys. Rev. B 1, 1449 (1970).

${ }^{35}$ S. Aksela, M. Pessa, and M. Karras, Z. Phys. 237, 381 (1970).

${ }^{36}$ J. P. Coad and J. C. Rivière, Z. Phys. 244, 19 (1971).

${ }^{37}$ J. P. Coad and J. C. Rivière, Surf. Sci. 25, 609 (1971).

${ }^{38}$ L. H. Jenkins and M. F. Chung, Surf. Sci. 24, 125 (1971).

${ }^{39}$ R. W. Fink, R. C. Jopson, H. Mark, and C. D. Swift, Rev. Mod. Phys. 38, 513 (1966).

${ }^{40}$ C. S. Fadley and D. A. Shirley, Phys. Rev. A 2, 1109 (1970)

${ }^{41}$ G. Wentzel, Z. Phys. 43, 524 (1927).

${ }^{42}$ A. E. S. Green, D. L. Sellin, and A. S. Zachor, Phys. Rev. 184, 1 (1969).

${ }^{43}$ V. O. Kostroun, M. H. Chen, and B. Crasemann, Phys. Rev. A 3, 533 (1971).

${ }^{44} \mathrm{M}$. H. Chen, Ph. D. thesis (University of Oregon, 1972) (unpublished).

${ }^{45}$ M. H. Chen and B. Crasemann, in Ref. 10, p. 43.

${ }^{46}$ F. Herman and S. Skillman, Atomic Structure Calculations (Prentice-Hall, Englewood Cliffs, N. J., 1963).

${ }^{47}$ E. J. Callan, Phys. Rev. 124, 793 (1961).

${ }^{48}$ E. J. Callan, in Role of Atomic Electrons in Nuclear Transformations (Nuclear Energy Information Center, Warsaw, 1963), Vol. 3, p. 419.

\title{
Lower Bound to Limiting Fields in Nonlinear Electrodynamics*
}

\author{
Gerhard Soff, Johann Rafelski, and Walter Greiner \\ Institut für Theoretische Physik der Universität Frankfurt, Frankfurt am Main, Germany
}

(Received 31 August 1972)

\begin{abstract}
In view of new high-precision experiments in atomic physics it seems necessary to reexamine nonlinear theories of electrodynamics. The precise calculation of electronic and muonic atomic energies has been used to determine the possible size of the upper limit $E_{\max }$ to the electric field strength, which has been assumed to be a parameter. This is opposed to Born's idea of a purely electromagnetic origin of the electron's mass which determines $E_{\max }$. We find $E_{\max } \geq 1.7 \times 10^{20} \mathrm{~V} / \mathrm{cm}$.
\end{abstract}

In recent years nonlinear electrodynamics has not belonged to the mainstream of research because any effective work within this framework was handicapped by mathematical and numerical difficulties with nonlinear equations. As the nonlinear effects were expected to show up in experi- ments only when high electric fields of the order of $10^{18} \mathrm{~V} / \mathrm{cm}$ are involved, it was argued that the only possible experiments would be dominated by quantum effects, so that nonlinear effects would be invisible. ${ }^{1}$ However, very recent $\gamma$-spectroscopic experiments and calculations in atomic 
physics ${ }^{2-5}$ have been carried out with an accuracy of $0.005 \%$. Within this accuracy it is possible to check the validity of nonlinear electrodynamics to the same extent. Nonlinear electrodynamic Lagrangians which lead to an upper limit of the electric field strength were first introduced by Born. ${ }^{1,6}$ His special theory, as well as many other nonlinear electrodynamics, has the satisfactory property that the electromagnetic self-energy of the electron is finite and that the four-momentum of the electron deduced from the energy-momentum tensor is a Lorentz vector. ${ }^{6}$

Here we are mainly interested in the problem of electrostatic fields. Hence we will specialize to this case only $(\vec{B}=0)$. Our investigations will deal with a class of Hamiltonian densities $H^{(n)},{ }^{6}$

$$
H^{(n)}=\frac{E_{0}^{2}(n)}{2 n}\left[\left(1+\frac{D^{2}}{E_{0}^{2}(n)}\right)^{n}-1\right] \text {. }
$$

The parameter $n$ characterizes various theories. For example, $n=1$ yields Maxwell's theory, $n=\frac{1}{2}$ Born's original theory, and $n=0$ the Infeld-Hoffmann theory. ${ }^{6}$ These Hamiltonian densities reduce to Maxwellian form whenever $D^{2} \ll E_{0}^{2}$. Here $\overrightarrow{\mathrm{D}}$ is the electric displacement and is defined as $\vec{D}=-\partial L / \partial \vec{E} . \quad \vec{E}$ is the electric field strength and $L$ the Lagrangian density of the system. The latter is related to $H$ in electrostatics via the equation

$$
L=H-\overrightarrow{\mathrm{E}} \cdot \overrightarrow{\mathrm{D}} .
$$

The principle of superposition for the electric field strengths is no longer valid in nonlinear electrodynamics. However, as the electric displacement $\vec{D}$ obeys a linear Euler-Lagrange equation

$$
\vec{\nabla} \cdot \overrightarrow{\mathrm{D}}=4 \pi \rho,
$$

where $\rho$ is a given charge distribution, and since we can assume the superposition principle for these charges, it follows that the principle of superposition is also valid for the electric displacement $\vec{D}$. The interaction energy of a twoparticle system is then computed according to

$$
V=\int d^{3} x\left[H\left(\overrightarrow{\mathrm{D}}_{1}+\overrightarrow{\mathrm{D}}_{2}\right)-H\left(\overrightarrow{\mathrm{D}}_{1}\right)-H\left(\overrightarrow{\mathrm{D}}_{2}\right)\right] \text {. }
$$

$E_{0}$, which has not yet been specified, characterizes an upper limit $E_{\max }$ of the electric field strength, which exists whenever $n \leq 0.5$ in Eq. (1). Namely, from $\overrightarrow{\mathrm{E}}=\partial H / \partial \overrightarrow{\mathrm{D}}$ we obtain

$$
\overrightarrow{\mathrm{E}}=\overrightarrow{\mathrm{D}} \cdot\left[1+D^{2} / E_{0}^{2}(n)\right]^{n-1} \text {. }
$$

For a point charge $[\rho(r)=e \delta(r)]$ we have $\overrightarrow{\mathrm{D}}=e \overrightarrow{\mathrm{r}} / r^{3}$ and it follows straightforwardly that

$$
E_{\max }=E_{0}(n) \frac{(1-2 n)^{1 / 2-n}}{(2-2 n)^{1-n}} .
$$

In our earlier calculations ${ }^{7}$ we have used $E_{0}(n=0.5)$ $=1.2 \times 10^{18} \mathrm{~V} / \mathrm{cm}$, which has been calculated under the assumption that the total mass of the electron is of electromagnetic origin, ${ }^{1}$ that is,

$$
m_{e} c^{2}=\int d^{3} \times H^{(n)}=\frac{4 \pi E_{0}^{2}}{2 n} \int_{0}^{\infty} d r\left[\left(1+\frac{e^{2}}{r^{4} E_{0}^{2}}\right)^{n}-1\right] .
$$

Now, in applying the nonlinear theories to muonic atoms, one could consider the mass of the muon to be also of entirely electromagnetic origin. This would mean the $E_{\max }^{\text {muon }}$ would be about $(207)^{2}$ times larger. Clearly, such particle-dependent limiting field strengths cannot be ruled out $a$ priori, but such theories are unesthetic. It seems much more satisfactory to assume one universal limit to the field strength which is independent of the particle creating the field. ${ }^{8}$ Indeed, we will show that there is no necessity from present experiments to assume that the value of $E_{\max }$ is particle dependent, as mentioned above. The value of $E_{\max }$, which is not contradictory to the present accuracy of atomic ionization energies, will also be in agreement with muonic data.

Our calculations of transition energies of electronic and muonic atoms are carried out within the framework of the Dirac equation. The electrostatic potential is generated by a Fermi-type nuclear charge distribution. For simplicity the Thomas-Fermi model of the atom is used to determine the atomic charge distribution. This is good approximation for the inner shells which we are investigating here, where atomic shell effects play a minor role. We can compare our values of electronic eigenenergies with the results of Fricke $e t$ $a l .{ }^{5}$ and Freedman et al. ${ }^{4}$ : In the case of a Fermitype nuclear charge distribution of ${ }_{100} \mathrm{Fm}$ with a half-density radius $c=7.0 \mathrm{fm}$ and a surface thickness $t=2.2 \mathrm{fm}$, the Dirac-Fock self-consistent description ${ }^{5}$ gives $-142.929 \mathrm{keV}$ for the $1 \mathrm{~s}$ ionization energy; our result for the eigenenergy is $-142.920 \mathrm{keV}$. The difference of $9 \mathrm{eV}$ is equivalent to a change $\Delta c \approx 0.1 \mathrm{fm}$ of the nuclear radius, as pointed out by Fricke et al. ${ }^{5}$ The experimental value of Porter and Freedman ${ }^{2}$ is - 141. 963(13) $\mathrm{keV}$. The remaining difference of $\sim 1 \mathrm{keV}$ is attributed to several corrections. ${ }^{4,5}$

The largest nonlinear effects can be expected when the test particle, e.g., the electron or muon, moves in the high electric field of the nucleus, i. e., when the radial probability density has its maximum at the edge of the nucleus. Such a situation is realized for, example, in the case of the muonic $1 s$ eigenfunction in ${ }_{82} \mathrm{~Pb}$. The disadvantage of this case is the fact that the nuclear radius is adjusted from the $2 p-1 s$ transitions, which may hide the nonlinear electrodynamic effect. It can be easily shown from Eq. (4) that the nonlinear interaction energy differs from the Coulomb potential in first order by a term proportional to $r^{-5}$, the proportionality constant being independent of the 


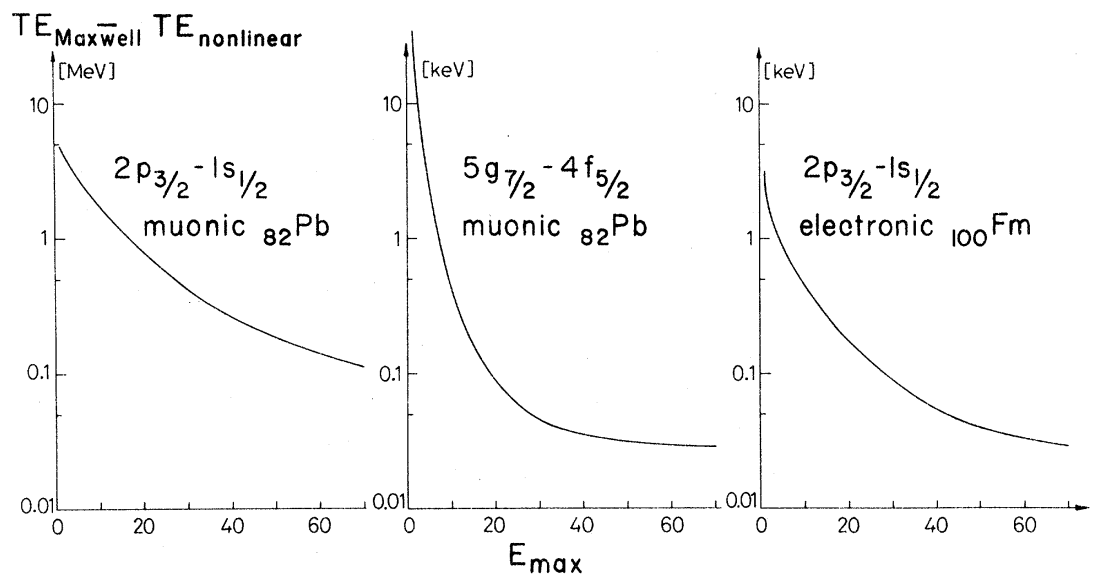

FIG. 1. Theoretical deviations caused by nonlinear theories of several transition energies $T E$ as function of the limiting field value $E_{\max }$ in units of $E_{B}=1.2 \times 10^{19} \mathrm{~V} / \mathrm{cm}$. shape of the nuclear charge distribution:

$$
V(r) \underset{r>c}{\approx}-\frac{Z e^{2}}{r}+\frac{Z^{3} e^{4}(1-n)}{E_{0}^{2}(n) 5} \frac{1}{r^{5}} \equiv V_{\text {Coul }}+V_{\text {add }} .
$$

Thus wave functions lying well outside of the nucleus, although less sensitive to nonlinear effects, may give a more decisive test of the nonlinear electrodynamics, since no unknown nuclear quantities. disturb the discussion. It is worthwhile to note here that nonlinear potential effects of type (8) can in a linear (Maxwell) theory always be simulated by a nuclear halo effect, since the additional potential of Eq. (8) corresponds to a "linear" charge distribution

$$
\rho_{\text {add }}=\frac{1}{4 \pi} \Delta V_{\text {add }}=\frac{Z^{3} e^{4} 4(1-n)}{4 \pi E_{0}^{2}(n)} \frac{1}{r^{7}} .
$$

The effect of nonlinearities is illustrated in Fig. 1, where the deviations $T E_{\text {Maxwel1 }}-T E_{\mathrm{BI}}$ of the transition energies of the Born-Infeld theory from Maxwell electrodynamics are shown as a function of the limiting field value $E_{\max } \cdot E_{\max }$ is denoted in units of $E_{B}=1.2 \times 10^{18} \mathrm{~V} / \mathrm{cm}$ as proposed by Born. The left-hand curve shows the muonic $2 p_{3 / 2}-1 s_{1 / 2}$ transition in ${ }_{82}^{208} \mathrm{~Pb}$. As expected, we observe here the largest absolute influence of nonlinearities, but it is also strongly dependent on the in principle unknown nuclear size. The middle curve shows the change of muonic $5 g_{7 / 2}-4 f_{5 / 2}$ transition in ${ }_{82}^{208} \mathrm{~Pb}$. We have chosen this particular outer transition because it has been measured very precisely by Dixit et al.$^{3}$ (see below). The curve on the right-hand side shows the electronic $2 p_{3 / 2}-1 s_{1 / 2}$ transition in ${ }_{100} \mathrm{Fm}$. In Fig. 2 the same results are shown in relative units; i.e., the deviation at $E_{\max }$ has been divided by its value at $E_{\max }=1$. It can be seen that the $2 p_{3 / 2}-1 s_{1 / 2}$ electronic transition in ${ }_{100} \mathrm{Fm}$ is most sensitive to nonlinearities apart from the muonic $2 p_{3 / 2}-1 s_{1 / 2}$ transition in ${ }_{82} \mathrm{~Pb}$ which, however, is uncertain due to large effects of the shape of nuclear charge distribution. We have quoted here only results for $n=0.5$ theory, because the calculations show that the Born-Infeld theory is indeed representative for the whole class of theories denoted in Eq. (1).

In muonic-atom experiments disagreement between theory [including finite size effects and vacuum polarization up to order $\left.(Z \alpha)^{3}\right]$ and experiment has recently been reported for higher transitions such as $5 g_{7 / 2}-4 f_{3 / 2}$ in ${ }^{208} \mathrm{~Pb} .{ }^{3,9}$ It is suggestive to try to explain these discrepancies by using nonlinear electrodynamics. It is important here that the over-all good agreement achieved for the various inner transitions within Maxwell electrodynamics is not destroyed. Therefore, we fit at first the $2 p_{3 / 2}-1 s_{1 / 2}$ transition as illustrated in Fig. 3 for a given limiting field $E_{0}$. The change of transition energies of $2 p_{3 / 2}-1 s_{1 / 2}$ in muonic ${ }^{208} \mathrm{~Pb}$ caused by nonlinear theories is shown as a func-

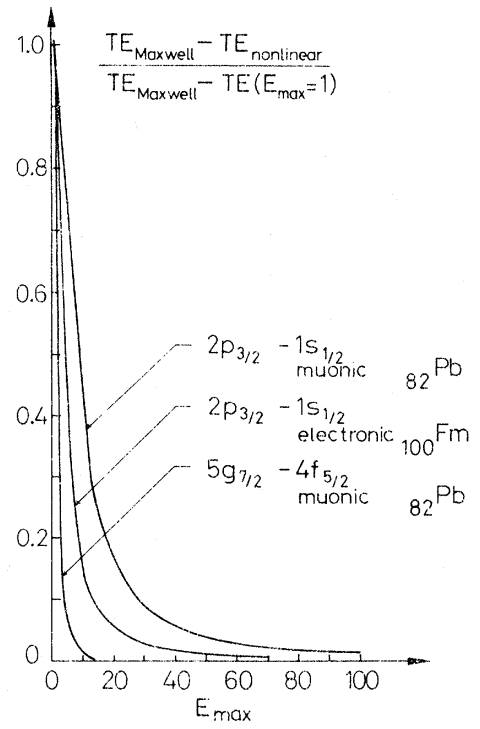

FIG. 2. Same deviations as in Fig. 1 in relative units. 


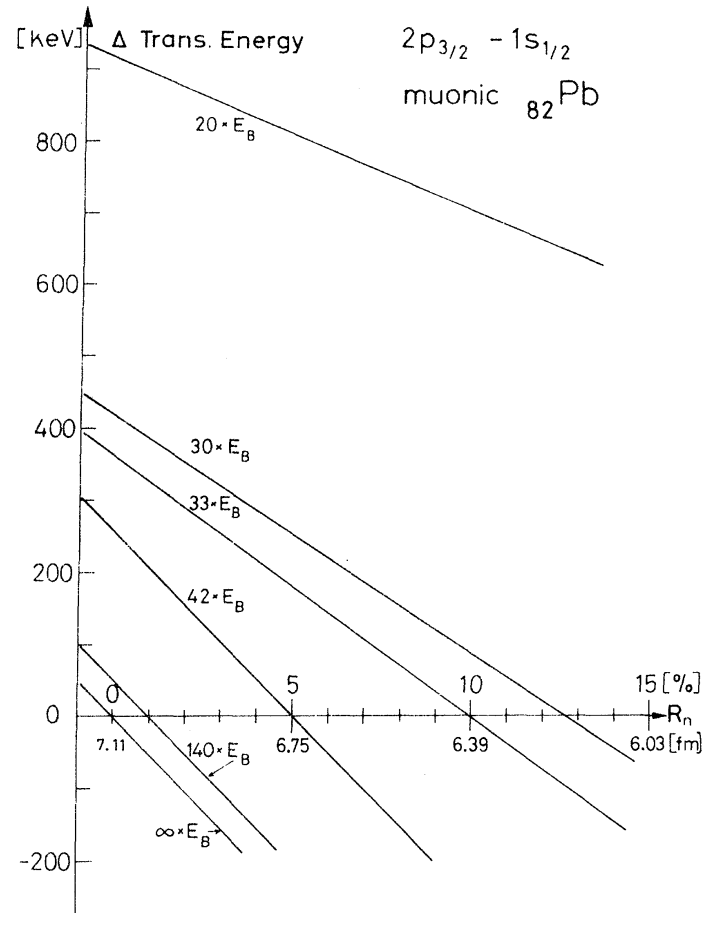

FIG. 3. Deviations in the transition energy of the muonic $2 p_{3} / 2-1 s_{1 / 2}$ transition in ${ }_{82} \mathrm{~Pb}$ as function of the equivalent nuclear radius $R_{n}$. Different curves belong to different values of limiting field.

tion of the nuclear radius and its change relative to the "linear" radius in percent. Different curves belong to different values of the limiting field. It can be seen, for example, that for $E_{\max }=140 E_{B}$ (respectively, $33 E_{B}$ ) the nonlinear field effects are invisible in the $2 p_{3 / 2}-1 s_{1 / 2}$ transition, if the nuclear radius is renormalized simultaneously by $1 \%$ (respectively, 10\%). Because of the mentioned long range of nonlinearities and its different $r$ dependence compared to finite size effects, there is still a contribution to the inner electronic and most other inner and outer muonic transition energies. For example, using $E_{\max }=33 E_{B}$ and simultaneously a nuclear radius renormalized by $10 \%$, one obtains a contribution of about $30 \mathrm{eV}$ to the electronic $2 p_{3 / 2^{-}}$ $1 s_{1 / 2}$ transition in ${ }_{100} \mathrm{Fm}$. In our opinion this deviation lies within the theoretical uncertainty of the present experiments ${ }^{4,5}$ (observe, for example, that in these references the values for self-energy differ by $27 \mathrm{eV}$ ). An even higher absolute influence of such calculations may be observed in the muonic $5 g_{7 / 2}-4 f_{5 / 2}$ transition in lead. As the $5 g_{7 / 2}$ and $4 f_{5 / 2}$ wave functions lie far outside the nucleus, the finite size effects are very small $(10 \mathrm{eV})$ and are almost unchanged by $10 \%$ renormalization of the nuclear radius. From Fig. 1 we obtain a contribution of $40 \mathrm{eV}$ to the transition energy. This is only one-third of the deviation which has been observed by Dixit et al. ${ }^{3}$ However, very recently similar experimental results were obtained by Walter et $a l .,{ }^{10}$ who use corrected values for the third-order vacuum polarization and observe a discrepancy between theory and experiment of the order of 60 $\pm 25 \mathrm{eV}$ for the muonic $5 g_{9 / 2}-4 f_{7 / 2}$ and $5 g_{7 / 2}-4 f_{5 / 2}$ transitions in ${ }_{80}^{\mathrm{nat}} \mathrm{Hg}$ and ${ }_{81}^{203} \mathrm{Tl}$. We note that after a contribution of $40 \mathrm{eV}$ for nonlinear electrodynamics has been included, it seems that agreement between theory and experiment could be achieved. This is also true for other experimental results given by Dixit et al., ${ }^{3}$ after the theoretical values of the vacu um polarization have been corrected as indicated by Sundaresan et al. ${ }^{11}$ However, when these inner $\left(2 p_{1 / 2} \rightarrow 1 s\right)$ and outer $\left(5 g_{7 / 2} \rightarrow 4 f_{5 / 2}\right)$ transitions were fitted to the nuclear radius $R_{n}$ and the limiting field $E_{\text {max }}$, no agreement within experimental errors was found with other well-known inner transitions: For example, the $2 s_{1 / 2}-2 p_{3 / 2}$ and $3 d_{5 / 2}-2 p_{3 / 2}$ transition energies are then predicted too small by 38 and 31 $\mathrm{keV}$, respectively. This big disagreement could only be substantially reduced when $E_{\max }$ was increased to about $E_{\max } \approx 140 E_{B} \approx 1.7 \times 10^{20} \mathrm{~V} / \mathrm{cm}$. This value has thus to be considered as a lower bound for $E_{\max }$ compatible with the high-precision experiments discussed here.

We mentioned before that nonlinear electrodynamics with renormalized nuclear charge radii are equivalent to the usual Maxwell theory using nuclear charge distributions with long tails [halo effect-see Eq. (8)]. Such a halo effect cannot be understood with nuclear wave functions, the tail of which is determined by the binding energy of the nucleons in the Fermi surface. The nonlinear effects, however, can obviously also not simulate such a nuclear halo, because of their smallness (large $E_{\max }$ ).

These lower bounds for the limiting electric field give only small effects when superheavy nuclei are considered; we find that the value of $Z_{\mathrm{cr}}{ }^{7,12}$ is shifted by no more than two units. Finally, we mention that the lower bound for $E_{\max }$ obtained here leads, of course, to no discrepancy for the Lamb-shift experiments. Already the much larger nonlinearities obtained with the smaller value $E_{\max }$ $=33 E_{B}=4 \times 10^{10} \mathrm{~V} / \mathrm{cm}$ lead to a correction to the Lamb shift in hydrogen atom of only $0.006 \mathrm{MHz}$, which is less than experimental and theoretical uncertainties.

We acknowledge interesting discussions and correspondence with B. Muller, Dr. M.S. Freedman, and Dr. B. Fricke.
*Work supported by the Deutsche Forschungsgemeinschaft, by. the Bundesministerium für Bildung und Wissenschaft, and by the
Gesellschaft für Schwerionenforschung (GSI).

${ }^{1}$ M. Born, Ann. Inst. Henri Poincare 7, 155 (1937) and 
references therein.

${ }^{2}$ F. T. Porter and M. S. Freedman, Phys. Rev. Lett. 27, 293 (1971).

${ }^{3}$ M. S. Dixit et al., Phys. Rev. Lett. 27, 878 (1971).

${ }^{4}$ M. S. Freedman, F. T. Porter, and J. B. Mann, Phys. Rev. Lett. 28, 711 (1972).

${ }^{5}$ B. Fricke, J. P. Declaux, and J. T. Waber, Phys. Rev. Lett. 28, 714 (1972)

${ }^{6} \mathrm{~J}$. Rafelski, L. P. Fulcher, and W. Greiner, Nuovo Cimento B 7, 137 (1972).

${ }^{7}$ J. Rafelski, L. P. Fulcher, and W. Greiner, Phys. Rev. Lett. 27, 958 (1971)

\author{
${ }^{8}$ This idea may be stated in the more general form by \\ postulating the existence of a universal limiting energy density for \\ all fields (strong, electromagnetic, weak, and gravitational). \\ ${ }^{9}$ G. A. Rinker, Jr. and M. Rich, Phys. Rev. Lett. 28, 640 \\ (1972). \\ ${ }^{10} \mathrm{H}$. K. Walter et al., Phys. Lett. B 40, 197 (1972); R. Engfer \\ (private communication). \\ ${ }^{11}$ M. K. Sundaresan and P. J. S. Watson, Phys. Rev. Lett. 29, \\ 15 (1972). \\ ${ }^{12}$ B. Müller, H. Peitz, J. Rafelski, and W. Greiner, Phys. Rev. \\ Lett. 28, 1235 (1972).
}

\title{
Relativistic Correction to the $1 S \rightarrow 2 S$ Transition in Hydrogen Atoms in Intense Fields
}

\author{
S. N. Haque
}

Centre of Advanced Study in Physics, University of Delhi, Delhi-7, India

(Received 22 November 1971; revised manuscript received 26 July 1972)

\begin{abstract}
Using a recent method due to Reiss, the relativistic effect to multiphoton absorption in hydrogenlike atoms in the presence of intense electromagnetic fields of long wavelength is calculated. For an intensity parameter less than unity the corrections are found to be small for any number of photons absorbed. However if the intensity parameter exceeds unity by a considerable amount, corrections become significant.
\end{abstract}

\section{INTRODUCTION}

With the discovery of the laser ${ }^{1}$ in the last decade, it has become possible to achieve photon beams extremely monochromatic and having very high intensity. With rapid technical development, the intensity has been multiplied by several orders of magnitude so that processes involving stimulated emission or absorption of several photons from hydrogenlike atoms can now be ol. srved in the laboratory. The essential nonlinear character of the intensified interaction with matter leads to serious difficulty in the application of the perturbation theory. To avoid this, Reiss ${ }^{2}$ developed an approximation technique for the treatment of boundquantum systems in the presence of an intense electromagnetic field of long wavelength. A treatment of free-quantum systems in the presence of an intense external electromagnetic field has been given by Volkov and others. ${ }^{3}$ Approximate calculations for the multiphoton ionization of hydrogen and inert gas atoms have been made by Bebb and Gold, ${ }^{4}$ while Zernik ${ }^{5}$ has performed an exact calculation for the double-photon ionization of the metastable $2 S$ level of hydrogen. The exact method of Zernik has been generalized for the absorption of any number of photons by Gontier and Trahin, ${ }^{6}$ who have also given numerical results. All these perturbation treatments suffer from the drawback that their results are valid for fluxes less than $10^{33}$ photons $/ \mathrm{cm}^{2} \mathrm{sec}$, where ionization cross sections for many-photon processes are generally very much smaller than for single-photon process. The approach of Reiss consists of applying a unitary transformation to the semiclassical matter field which approximately removes the electromagnetic field from the problem. The accuracy of this technique increases with the number of phtons involved. For long wavelength and interactions involving a large number of photons, the transition amplitude has a simple form similar to the first-order perturbation result. Explicit nonlinear effects appear in the intensity-dependent deviation of the transition probability from the power-law dependence of the intensity. As an example of this method, Reiss has calculated stimulated emission together with a weak Raman photon from an excited state of the hydrogen atom and has shown how the perturbation approach breaks down in this case. ${ }^{7}$ Most of these applications of Reiss's technique are confined to nonrelativistic (NR) cases only. The smallness of relativistic terms in the perturbation approach does not necessarily imply the corresponding smallness for photon intensity, $>10^{33} / \mathrm{cm}^{2} \mathrm{sec}$, especially as higher-order processes contribute significantly to the transition amplitude.

The purpose of this paper is to see how high intensity magnifies relativistic effects. As an example, bound-bound transitions in hydrogenlike atoms are calculated. We have only considered here the $1 S \rightarrow 2 S$ transition owing to mulitphoton absorption.

In Sec. II we have proved the commutator theorem of Reiss for relativistic Hamiltonian and have 